\title{
Smoothing of interfaces in ultrathin Mo/Si multilayers by ion bombardment
}

\author{
A. Kloidt, H. J. Stock, U. Kleineberg, T. Döhring, M. Pröpper, \\ B. Schmiedeskamp and U. Heinzmann \\ Universität Bielefeld, Fakultät für Physik, W-4800 Bielefeld (Germany)
}

\begin{abstract}
Mo/Si multilayers with a bilayer thickness of $2.6 \mathrm{~nm}$ are produced by electron beam evaporation in ultrahigh vacuum for soft $\mathrm{X}$-ray optical applications. High reflectivities resulting from constructive interference in the stack are limited by the optical constants of the materials and by the quality of the interfaces. Smoothing of the boundaries is obtained by bombardment of the deposited layers with $\mathrm{Ar}^{+}$ions. The smoothness of the interfaces is controlled during the deposition by in situ measurement of the reflectivity for the $\mathrm{C} \mathrm{K}$ radiation of the stack and after completion of the stack by means of a grazing $\mathrm{X}$-ray reflection set-up with $\mathrm{Cu} \mathrm{K} \alpha$ radiation. The soft $\mathrm{X}$-ray reflectivity is measured with a laser-induced plasma light source.
\end{abstract}

\section{Introduction}

For the wavelength range above the $\mathrm{Si} \mathrm{L}$ absorption edge $(\lambda=13-20 \mathrm{~nm})$ normal incidence $\mathrm{Mo} / \mathrm{Si}$ soft $\mathrm{X}$ ray mirrors with a bilayer thickness $d$ of $7-10 \mathrm{~nm}$ are produced by sputtering [1-5] as well as by electron beam evaporation $[6,7]$. These multilayers have reflectivities of more than $50 \%$. The interfacial roughness $\sigma$ in these multilayers is typically $0.5 \mathrm{~nm}$ and hardly affects the reflectivity. The material combination $\mathrm{Mo} / \mathrm{Si}$ is also interesting for X-ray optical applications at shorter wavelengths, e.g. smaller than $4.4 \mathrm{~nm}$ (C K edge). In this case multilayers with small $d$ values $(d<3 \mathrm{~nm})$ are desired. The upper limit for achievable reflectivities is then smaller owing to the less favourable optical constants of the two materials and to roughness and intermixing at the interfaces (which is more significant for layer systems with smaller $d$ spacings). In this work we have fabricated multilayer systems with a period of $2.6 \mathrm{~nm}$ as near normal incidence soft X-ray mirrors. A smoothing of the interfaces for such an ultrathin multilayer system is attempted by ion bombardment with $\mathrm{Ar}^{+}$ions and thermal treatment of the substrate during the deposition run. The effect on smoothing the boundaries is obtained by in situ measurements of the reflection of $\mathrm{C} \mathrm{K}$ radiation vs. multilayer thickness and ex situ grazing X-ray reflection at the $\mathrm{Cu} \mathrm{K} \alpha$ line. In previous works [8-11] ion polishing has been applied successfully. Recently Schlatmann et al. reported on an improvement of reflectivity by ion beam etching of $\mathrm{Mo} / \mathrm{Si}$ multilayer coatings [12]. The effect on smoothing the surface discussed in our work is in agreement with these results. Beyond this we present reflectivity measurements in the soft $\mathrm{X}$-ray region for which the multilayers are designed.

\section{Experiments and results}

The deposition procedure is identical with that of the normal incidence mirrors for the $100 \mathrm{eV}$ region with a bilayer thickness of about $7 \mathrm{~nm}$. Details of the deposition system are described in refs. 6 and 13; it is completed by an ion gun at a distance of $20 \mathrm{~cm}$ to the substrate and a grazing angle of $20^{\circ}$. The incidence angle $\alpha$ of the $\mathrm{C} \mathrm{K}$ radiation for the in situ reflectivity measurement is now $30^{\circ}$ with respect to the surface normal. This results in a multilayer mirror with a period of $2.6 \mathrm{~nm}$, if the evaporation material is changed at the maxima and minima of the $\mathrm{C} \mathrm{K}$ reflectivity curve. The multilayer coatings are deposited onto $\mathrm{Si}(111)$ wafers covered with natural oxide.

Smoothing of the interfaces in the $7 \mathrm{~nm}$ stacks was carried out by thermal treatments during deposition $[6,7]$ and yielded the smoothest interfaces for a deposition temperature of $150^{\circ} \mathrm{C}$. Firstly these deposition conditions are directly transferred to the ultrathin layers: a $\mathrm{C} \mathrm{K}$ reflectivity curve obtained during deposition of 22 bilayers $(N=22)$ and at a deposition temperature of $150^{\circ} \mathrm{C}$ is given in Fig. 1(a). The measured average reflectivity and amplitude of the oscillation during growth increase at first and decrease after a few periods. After 22 bilayers it is impossible to determine extremes to change the evaporating material. The reason for this behaviour of the reflectivity curve is the accumulation of roughness at the boundaries during multilayer depo- 


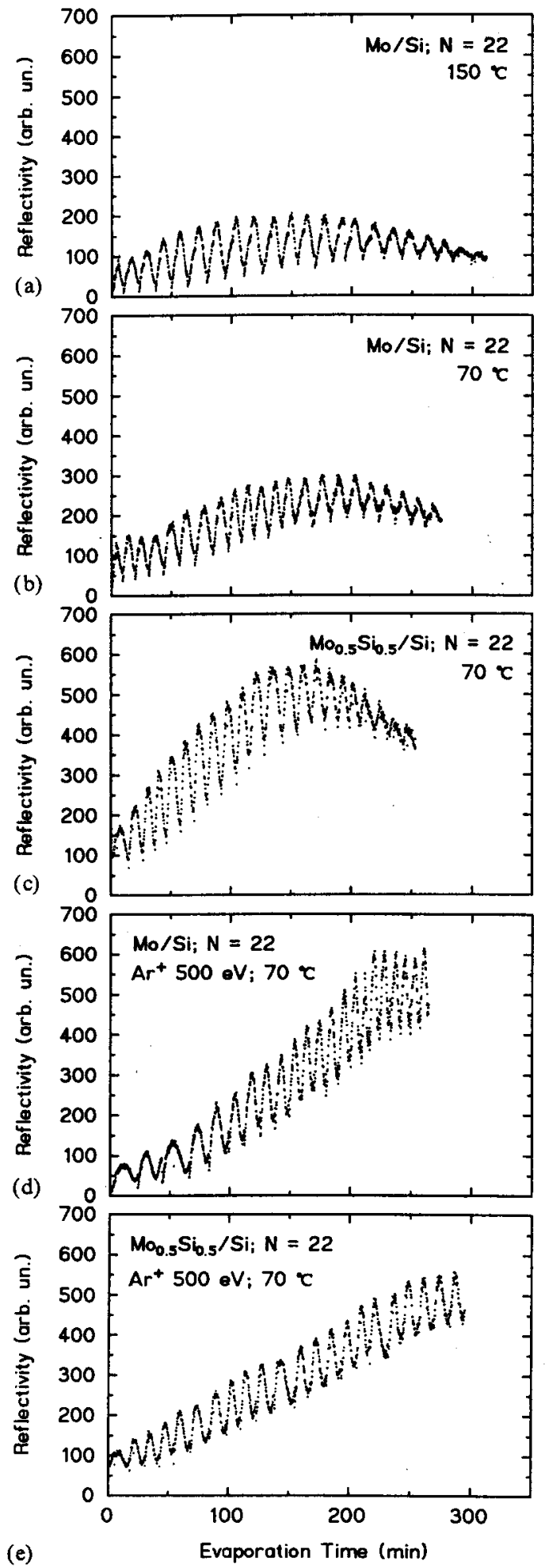

Fig. 1. In situ reflectivity of $\mathrm{C} \mathrm{K}$ radiation (angle of incidence $\alpha=30^{\circ}$ ) during deposition of multilayers under different processing conditions.

sition. This roughness increases with multilayer thickness, in contrast to the $7 \mathrm{~nm}$ stacks where no increase in the roughness was observed for deposition at $150^{\circ} \mathrm{C}$.
In order to find more favourable deposition conditions for the $2.6 \mathrm{~nm}$ stacks we discuss models for the deposition and growth process. According to Niibe et al. [2] thermal treatment of the multilayer stack stimulates surface diffusion. This will suppress the growth of columnar structures in the multilayers. The interfaces of the stack are smooth. For the $2.6 \mathrm{~nm}$ stacks we find rough interfaces at a substrate temperature of $150{ }^{\circ} \mathrm{C}$. Deposition at a substrate temperature of $70^{\circ} \mathrm{C}$ yields a slightly better result which is illustrated by the in situ reflectivity curve of Fig. 1(b). This can be explained by initial growth of islands. The average diameter of islands may be larger at a higher substrate temperature $\left(150^{\circ} \mathrm{C}\right)$ because of higher surface diffusion and probably does not yield a closed film in the early stage of film growth. This contributes to the interface roughness (the absorber layer thickness in our $2.6 \mathrm{~nm}$ stacks is only about $1 \mathrm{~nm}$ ).

Further, the formation of interfacial layers in the system $\mathrm{Mo} / \mathrm{Si}$ is well known [6,14-16]. Slaughter et al. [14] proposed the model that this reaction is due to $\mathrm{Si}$ diffusion into the Mo layer during growth. Therefore we performed an experiment in which Mo and Si were mixed by simultaneous evaporation with a ratio of unity, in order to reduce the diffusion of $\mathrm{Si}$ into the absorber layer which results in a thickness decrease of the Si spacer layer. This mixing of Mo and $\mathrm{Si}$ results in an increase in the average reflectivity of the stack (Fig. 1(c)) but it does not decrease the interfacial roughness: the oscillations in the reflectivity curve (Fig. 1(c)) are again almost completely damped after deposition of 22 periods.

A considerable improvement in quality of the interfaces is obtained by bombardment with $\mathrm{Ar}^{+}$ions. Best results are achieved by a bombardment after every deposited Si layer with ion energies of $500 \mathrm{eV}$ and a current density lower than $1 \mu \mathrm{A} \mathrm{cm}^{-2}$. The exposure time is typically $10 \mathrm{~min}$. The effect on smoothing the interfaces can be seen in Fig. 1, if the in situ reflectivity curves with and without $\mathrm{Ar}^{+}$polishing are compared. After $\mathrm{Ar}^{+}$bombardment both reflected intensity and the amplitude of the oscillation are increased and indicate a better quality of the stack than at a deposition procedure without $\mathrm{Ar}^{+}$bombardment. This is valid for evaporation of Mo as well as for simultaneous evaporation of $\mathrm{Mo}$ and $\mathrm{Si}$ in the absorber layer. The in situ reflectivity during ion bombardment implies a decrease in the spacer layer thickness. So we evaporate $\mathrm{Si}$ to a minimum of the in situ reflectivity plus a thickness of a few tenths of a nanometer to be removed (see Figs. 1(d) and $1(\mathrm{e})$; the reflectivity during ion bombardment is not shown). The remaining surface is smooth for the next deposition step. We attribute the smoothing of the $\mathrm{Si}$ surface by sputtering to the removal of spikes. The process is surface sensitive and the energy of the 

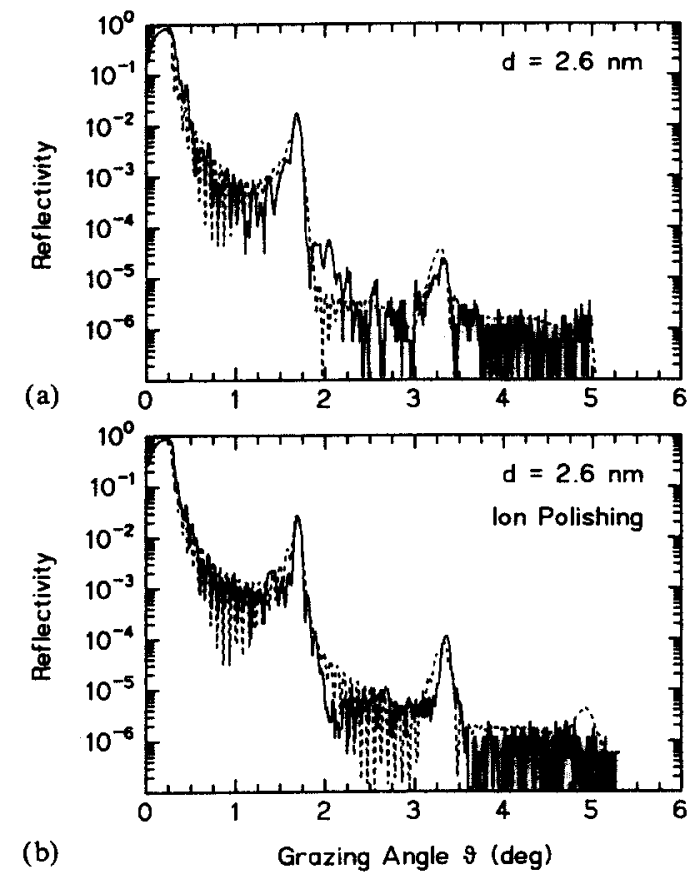

Fig. 2. Reflectivity measurements (- - ) and calculations (-.-) of $\mathrm{Cu}$ $\mathrm{K} \alpha$ radiation $(\lambda=0.154 \mathrm{~nm})$ at grazing angle for the same two mirrors presented in Figs. 1(c) and 1(e): (a) no ion polishing; (b) the intensity of the second Bragg order is significantly higher in the case with ion polishing.

$\mathrm{Ar}^{+}$ions is not large enough to destroy the underlying layers.

In addition to the in situ reflectivity measurement, the completed multilayers are characterized by ex situ grazing incidence $\mathrm{X}$-ray reflection at the $\mathrm{Cu} \mathrm{K} \alpha$ line. The reflectivity curve also gives information about the interfacial roughness in the stack. Figure 2 shows measurements (full line) of the same two multilayers as in Figs. 1(c) and 1(e). The sample with ion bombardment (Fig. 2(b)) exhibits a significantly higher intensity in the second Bragg order compared with that without (Fig. 2(a)). Parallel to the experiments we simulate the reflectivity $v s$. multilayer thickness and $v s$. grazing angle by calculations. Roughness at an interface reduces the reflected amplitude $r$. The ratio of the reflected amplitude $r_{0}$ of an ideal interface can be described by the Debye-Waller factor $\left(r=r_{0} \exp \left[-2(2 \pi \sigma \cos \alpha / \lambda)^{2}\right]\right)$. The in situ reflectivity curve is calculated with a linear and an exponential increase $\Delta \sigma$ in roughness at each interface as an approximation for the accumulation of roughness throughout the stack. Comparing measurements and calculations (not shown) we prefer an exponential increase in roughness for the substrate $\sigma=\sigma_{0}$ to $\sigma=\sigma_{0} \exp (\Delta \sigma N / \mathrm{nm})$ at the top of the stack. The calculated reflectivity $v s$. grazing angle with different large increases of roughness $\Delta \sigma$ at each interface is shown in Fig. 2 (broken lines). The calculation in Fig. 2(b) was

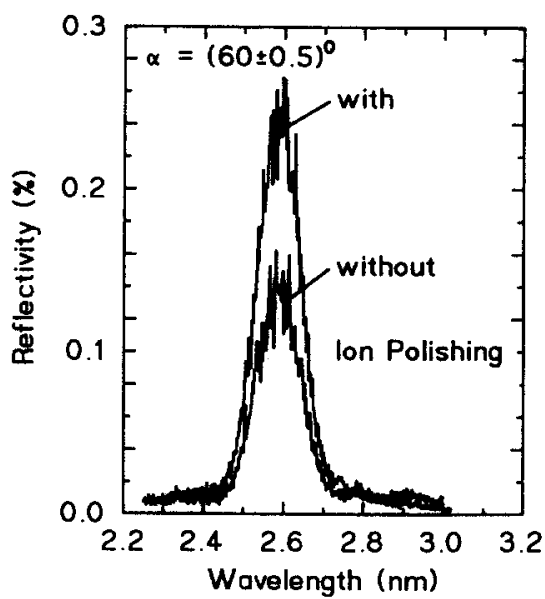

Fig. 3. Reflectivities of LIP radiation vs. wavelength for two soft $\mathrm{X}$-ray mirrors produced with and without ion bombardment of the interfaces (cf. Figs. 1(c) and 1(e)).

carried out with an increase in roughness from $\sigma_{0}=0.2 \mathrm{~nm}$ at the bottom of the stack to $\sigma=0.4 \mathrm{~nm}$ at the top. For deposition without $\mathrm{Ar}^{+}$polishing (Fig. 2(a)) the calculation is performed with an increase $\sigma-\sigma_{0}$ in roughness of $0.6 \mathrm{~nm}$. These parameters result also in good fits (not shown) for the in situ measurements of Fig. 1.

For the soft X-ray region reflectivity measurements of the X-ray mirrors are gained by using a laser-induced plasma light source (LIP) [17]. Figure 3 shows the reflectivity (for unpolarized LIP radiation) of the two soft X-ray mirrors ( $c f$. Figs. 1(c), 1(e) and 2) produced under the same deposition conditions except for ion polishing of the interfaces. The reflectivity of the multilayer with ion bombardment is significantly higher than that without. The reflectivity peaks measured at an angle $\alpha=60^{\circ}$ with respect to the surface normal lie in the "water window" $(2.4-4.4 \mathrm{~nm})$. The reflectivity values in the peak are considerably lower than those expected for an ideal multilayer system of $\mathrm{Mo}$ and $\mathrm{Si}$ $(\sigma=0 \mathrm{~nm})$, for which a calculation using the optical constants of Henke and coworkers [18] results in 4.3\% for s-polarized light. The increase in roughness from $0.2 \mathrm{~nm}$ at the bottom to $0.4 \mathrm{~nm}$ at the top of the stack reduces this value by almost a factor of 2 . Intermixing of $\mathrm{Mo}$ and $\mathrm{Si}$ in the absorber layers results in a reduction by a further factor of 2 . These intermixing processes occur obviously also if Mo is deposited as an absorber material. For the deposition in Fig. 1(d) we obtain a reflectivity value at $2.6 \mathrm{~nm}$ which lies between the two curves in Fig. 3. In addition the measurements are performed with unpolarized radiation, which results in a third reduction factor of almost 2 , since the $p$ component of the incident radiation is hardly reflected at an incidence angle of $60^{\circ}$. 


\section{Conclusion}

Mo/Si multilayers with a bilayer thickness of $2.6 \mathrm{~nm}$ are produced by electron beam evaporation. From the in situ reflectivity curve we obtained an accumulation of interfacial roughness throughout the stack. From a comparison with calculations we find an exponential increase in this roughness. This behaviour can be lessened by applying ion bombardment to the surface of every deposited Si layer. A smoothing of the interfaces is the result of the ion bombardment. The measured average reflectivity during deposition is drastically enhanced. This is in accordance with ex situ studies measuring the reflectivity of $\mathrm{Cu} K \alpha$ radiation at grazing angle and of LIP radiation $v s$. wavelength. The reflectivity in the soft X-ray region of a multilayer fabricated by using ion bombardment is enhanced by a factor of 2 . Altogether we obtain the result that ion bombardment results in a considerable smoothing of the interfaces in ultrathin $\mathrm{Mo} / \mathrm{Si}$ multilayers and an increase in the reflectivity. The achievable reflectivity values are, however, still limited by a slight increase in roughness and an intermixing of Mo and $\mathrm{Si}$.

\section{Acknowledgments}

Financial support by BMFT (13N 5539 and 5994) and Fa. Carl Zeiss, Oberkochen, is gratefully acknowledged.

\section{References}

1 S. Ogura, M. Niibe, Y. Watanabe, M. Hayashida and T. lizuka, Proc. Soc. Photo-Opt. Instrum. Eng., 984 (1988) 140.
2 M. Niibe, M. Hayashida, T. Iizuka, A. Miyake, Y. Watanabe, R. Takahashi and Y. Fukuda, Proc. Soc. Photo-Opt. Instrum. Eng., 1343 (1990) 2.

3 J. A. Trail, R. L. Byer and T. W. Barbee, Appl. Phys. Lett., 52 (4) (1988) 269.

4 N. M. Ceglio, D. P. Gaines, D. G. Stearns and A. M. Hawryluk, Opt. Commun., 69 (1989) 285.

5 D. G. Stearns, R. S. Rosen and S. P. Vernon, J. Vac. Sci. Technol. A, 9 (5) (1991) 2662

6 A. Kloidt, K. Nolting, U. Kleineberg, B. Schmiedeskamp, U. Heinzmann, P. Müller and M. Kühne, Appl. Phys. Lett., 58 (23) (1991) 2601.

7 A. Kloidt, H. J. Stock, K. Nolting, U. Kleineberg, B. Schmiedeskamp, U. Heinzmann, P. Müller and M. Krumrey, TATF91 Conf. Proc., in Vide, Couches Minces, 259 (1991) 173.

8 E. Spiller, Appl. Phys. Lett., 54 (23) (1989) 2293.

9 E. Spiller, Opt. Eng., 29 (6) (1990) 609.

10 E. J. Puik, M. J. van der Wiel, H. Zeijlemaker and J. Verhoeven, Appl. Surf. Sci., 47 (1991) 63.

11 E. J. Puik, M. J. van der Wiel, H. Zeijlemaker and J. Verhoeven, Appl. Surf. Sci., 47 (1991) 251.

12 R. Schlatmann, C. Lu, J. Verhoeven, E. J. Puik and M. J. van der Wiel, in Physics of X-ray Multilayer Structures Technical Digest 1992, Vol. 7, Optical Society of America, Washington, DC, 1992, p. PD6-1.

13 B. Schmiedeskamp, B. Heidemann, U. Kleineberg, A. Kloidt, M. Kühne, H. Müller, P. Müller, K. Nolting and U. Heinzmann, Proc. Soc. Photo-Opt. Instrum. Eng., 1343 (1990) 64.

14 J. M. Slaughter, P. A. Kearney, D. W. Schulze and C. M. Falco, Proc. Soc. Photo-Opt. Instrum. Eng., 1343 (1990) 73.

15 P. Boher, Ph. Houdy, L. Hennet, M. Kühne, P. Müller, J. P. Frontier, P. Trouslard, C. Senillou, J. C. Joud and P. Ruterana, Proc. Soc. Photo-Opt. Instrum. Eng., 1547 (1991) 21.

16 R. S. Rosen, M. A. Viliardos, M. E. Kassner, D. G. Sterns and S. P. Vernon, Proc. Soc. Photo-Opt. Instrum. Eng., 1547 (1991) 212.

17 N. Böwering, T. Döhring, U. Gärner and U. Heinzmann, Laser Part. Beams, 9 (2) (1991) 593.

18 B. L. Henke, E. M. Gullikson, J. C. Davis, M. Fryer and A. Oren, Proc. Soc. Photo-Opt. Instrum. Eng., 911 (1988) 107.

B. L. Henke, J. C. Davis, E. M. Gullikson and R. C. C. Perera, Center for X-Ray Optics, Lawrence Berkeley Laboratory, 1988, data tabulation in disk form provided by E. M. Gullikson. 